\title{
Numerical Simulation of Inert Solid-Phase Three- Component Mixtures Subjected to Explosive Compaction
}

\author{
Sergey Zelepugin, Oksana Ivanova \\ Tomsk Scientific Center of SB RAS \\ Tomsk, Russia \\ szel@dsm.tsc.ru, bliz3@yandex.ru
}

\author{
Aleksandr Yunoshev \\ Lavrentyev Institute of Hydrodynamics of SB RAS \\ Novosibirsk, Russia
}

\begin{abstract}
The article considers the axisymmetric problem concerning the explosive compaction of a mixture from aluminum, sulfur and carbon placed into a cylindrical steel ampoule. The inert substance (graphite) is added to the mixture to avoid the reaction between aluminum and sulfur. The numerical computations have demonstrated that the thickness of the explosive layer essentially influences on the final result of explosive compaction. Insufficient thickness of explosives, as well as the excessive thickness may be a reason for an incompletely compacted final product or lead to the formation of cracks or damage.
\end{abstract}

Keywords- shock wave synthesis; solid mixtures; multicomponent medium

\section{INTRODUCTION}

Producing and using new advanced materials are often connected with extreme conditions such as fast processes, high pressures and temperatures [1-5]. These processes are accompanied by structural changes and chemical reactions. At present, explosive technologies are commonly used in metal processing, including the technologies of shaping, welding, cutting, hardening, and compaction. Many of these technologies are already used in production, while the application of explosive compaction is restricted due to lack of ways to control and manage this process, and has not yet reached the level of technology so far. Many theoretical questions of explosive compaction of solid inert multicomponent mixtures must be answered.

This is caused by the complexity of studying the process of explosive compaction and inability to obtain reliable data on the dynamics of this phenomenon using final results of the experiments. The analysis of this process requires developing mathematical models, including the introduction of additional parameters and equations. There is also a need to take into account the initial parameters and characteristics of components in the mixture, and develop appropriate computational algorithms as well. This approach, together with the available experimental data, is to extend the range of application for numerical models and provide an opportunity to obtain reliable information on the mechanisms and dynamics of structural changes and ways to produce new advanced materials.

The aim of this paper is a study and numerical simulation of aluminum-sulfur-carbon mixtures under explosive compaction, taking into account the thickness of the explosive layer on the basis of a multicomponent medium model [6].

\section{FORMULATION OF THE PROBLEM}

The system of equations describing the nonstationary adiabatic motion of each component in a solid inert compressible mixture comprises the equations of continuity (Eq. 1), momentum (Eq. 2), and energy (Eq. 3) [6, 7]:

$$
\begin{gathered}
\frac{\partial}{\partial t}\left(\alpha_{i} \rho_{i}\right)+\nabla \alpha_{i} \rho_{i} v_{i}=0,(i=1,2, \ldots, N), \\
\alpha_{\imath} \rho_{i} \frac{d_{i} \mathrm{v}_{i}}{d t}=\nabla \sigma_{i}+\alpha_{i} \sum_{j=l}^{N} \alpha_{j} \mathbf{R}_{j i}, \quad(i=1,2, \ldots, N), \\
\alpha_{i} \rho_{i} \frac{d_{i} E_{i}}{d t}=\sigma_{i} \varepsilon_{i}+\alpha_{i} \sum_{j=1}^{N} \alpha_{j} \Phi_{j i},(i=1,2, \ldots, N), \\
\text { where } \frac{d_{i}}{d t} \equiv \frac{\partial}{\partial t}+v_{i}^{k} \frac{\partial}{\partial x^{k}} .
\end{gathered}
$$

Here $t$ is the time; $\rho_{i}$ is the density of the $i$-th component equal to the mass of $i$-th component per unit volume of the $i$-th component; $v_{i}$ is the velocity vector; $E_{i}$ is the internal specific energy; $\varepsilon_{i}$ is the strain rate tensor; $\sigma_{i}=-P_{i} \delta_{i}+S_{i}$ is the stress tensor; $P_{i}$ is pressure; $S_{i}$ is the stress deviator; $R_{j i}$ is the intensity of the momentum exchange between the $j$-th and $i$-th components; $\Phi_{j i}$ is the intensity of the energy exchange between the $j$-th and $i$-th components; $N$ is the number of components. 
Volume fractions of the mixture occupied by each component [7] are given by:

$$
\alpha_{1}+\alpha_{2}+\ldots+\alpha_{N}=1,\left(\alpha_{i} \geq 0\right), \alpha_{i}=\rho_{i}^{*} / \rho_{i}
$$

where $\rho_{i}^{*}$ is the reduced density (mass of the $i$-th component per unit volume).

To solve the explosive compaction problems of porous mixtures, the finite element method is used. On the basis of this method every component of a mixture simultaneously occupies the same volume as the mixture and consists of a set of the final elements connected with the node points. Inside every element, components interact with each other, exchanging momentum and energy within the framework of the multicomponent medium model. After interaction of components and their summary contribution to node forces of an element, the components in a mixture obtain the velocity of the corresponding element.

Evolution of porosity in the material (compression and growth of pores) is simulated using a kinetic model of the active type, which determines changes in specific volume of pores influencing on the material properties and causing stress relaxation [8]:

$$
\frac{d V_{f i}}{d t}=\left\{\begin{array}{l}
0, \text { if }\left|P_{s i}\right| \leq P_{i}^{*} \text { or }\left(P_{s i}>P_{i}^{*} \text { and } V_{f i}=0\right) \\
-\operatorname{sign}\left(P_{s i}\right) K_{f i}\left(\left|P_{s i}\right|-P_{i}^{*}\right)\left(V_{2 i}+V_{f i}\right), \\
\text { if } P_{s i}<-P_{i}^{*} \text { or } \quad\left(P_{s i}>P_{i}^{*} \text { and } V_{f i}>0\right),
\end{array}\right.
$$

where $P_{i}^{*}=P_{k i} V_{1 i} /\left(V_{f i}+V_{1 i}\right), P_{s i}$ is the pressure in the solid (undamaged) part of the $i$-th component in the mixture, $V_{l i}$, $V_{2 i}, P_{k i}, K_{f i}$ are the experimentally determined constants of the material.

Pressure in the undamaged component of the mixture is a function of specific volume and internal energy, and over the entire range of loading conditions, it is determined by the MieGrüneisen equation of state according to the formula [9]:

$$
\begin{aligned}
& P_{i}=\rho_{0_{i}} u_{c_{i}}{ }^{2} \mu_{i}+\rho_{0_{i}} u_{c i}{ }^{2}\left[1-\gamma_{0_{i}} / 2+2\left(b_{i}-1\right)\right] \mu_{i}{ }^{2}+ \\
& \rho_{0_{i}} u_{c_{i}}{ }^{2}\left[2\left(1-\gamma_{0_{i}} / 2\right)\left(b_{i}-1\right)+3\left(b_{i}-1\right)^{2}\right] \mu_{i}{ }^{3}+\gamma_{0_{i}} \rho_{0_{i}} E_{i}
\end{aligned}
$$

Here $\mu_{i}=V_{0_{i}} /\left(V_{i}-V_{f_{i}}\right)^{\prime}-1, \quad \gamma_{0_{i}}$ is the Grüneisen coefficient, $V_{0_{i}}$ and $V_{i}$ are the initial and current specific volumes, respectively, and $u_{c_{i}}$ and $b_{i}$ are the constants of the Hugoniot shock adiabat described by the relation:

$$
u_{s_{i}}=u_{c_{i}}+b_{i} u_{p_{i}}
$$

where $u_{s_{i}}$ is the shock wave velocity and $u_{p_{i}}$ is the particle velocity of a component behind the shock wave front.
Studying the deformation of multicomponent media, it is necessary to take into account the state and response of each component, as well as, in contrast to a homogeneous mixture, not only the displacement of the external boundaries of the selected volume, but also the displacement of components in the selected volume of the mixture. Here, we consider the equal pressures of the components to be a condition for joint deformation of components during the interaction in the mixture, which determines volume concentrations of the components [9]:

$$
P=P_{i}\left(V_{i}, E_{i}\right)=P_{j}\left(V_{j}, E_{j}\right)=\ldots=P_{N}\left(V_{N}, E_{N}\right) .
$$

The change in porosity is assumed to be influenced only by the spherical stress component or pressure, whereas the components of the stress deviator are bounded by the independent deviatoric yield function [8]:

$$
2 G_{i}\left(\varepsilon_{i_{k j}}-\frac{1}{3} \varepsilon_{i_{q q}} \delta_{i_{k j}}\right)=\frac{d S_{i_{k j}}^{0}}{d t}+\lambda_{i} S_{i_{k j}},
$$

where $d S_{i_{k j}}^{0} / d t$ is the Jaumann derivative given by:

$$
\frac{d S_{i_{k j}}^{0}}{d t}=\frac{d S_{i_{k j}}}{d t}-S_{i_{k q}} W_{i_{j q}}-S_{i_{j q}} W_{i_{k q}}
$$

and $2 W_{i_{k j}}=\partial v_{i_{i}} / \partial x_{j}-\partial v_{i_{j}} / \partial x_{i}$. Parameter $\lambda$ is zero for elastic deformation, and for plastic deformation, it is determined from the Mises yield criterion [8]:

$$
S_{i_{k j}} S_{i_{k j}}=\frac{2}{3} \sigma_{i}^{2}
$$

Here $G_{i}$ is the shear modulus and $\sigma_{\mathrm{i}}$ is the dynamic yield point:

$$
\begin{aligned}
& G_{i}=G_{0_{i}} K_{T_{i}}\left(1+\frac{c_{i} P_{i}}{\left(1+\mu_{i}\right)^{1 / 3}}\right) \frac{V_{3_{i}}}{\left(\mathrm{~V}_{f i}+V_{3_{i}}\right)}, \\
& \sigma_{i}=\left\{\begin{array}{l}
\sigma_{0_{i}} K_{T_{i}}\left(1+\frac{c_{i} P_{i}}{\left(1+\mu_{i}\right)^{1 / 3}}\right)\left(1-\frac{V_{f_{i}}}{V_{4_{i}}}\right), \text { if } V_{f_{i}} \leq V_{4_{i}}, \\
0, \text { if } V_{f_{i}}>V_{4_{i}}
\end{array},\right. \\
& K_{T_{i}}=\left\{\begin{array}{l}
1, \quad \text { if } \quad T_{0_{i}} \leq T_{i} \leq T_{1_{i}} \\
\frac{T_{m_{i}}-T_{i}}{T_{i_{m}}-T_{i_{1}}}, \text { if } T_{1_{i}}<T_{i}<T_{m_{i}} \\
0, \quad \text { if } \quad T_{i} \geq T_{m_{i}}
\end{array}\right.
\end{aligned}
$$

In the computations, the function $K_{T_{i}}\left(T_{i}\right)$ was chosen to model the nonthermal character of plastic deformation and 
dynamic strength of solids at high strain rates $\left(10^{4} \mathrm{~s}^{-1}\right.$ or higher).

The temperature was calculated using the following ratio:

$$
d T_{i}= \begin{cases}d\left(E_{i}-E_{0 x i}\right) / c_{p i}, & \text { if } T_{i}<T_{m i} \\ 0, & \text { if } T_{i}=T_{m i}, \\ d\left(E_{i}-E_{0 x i}-\Delta H_{m i}\right) / c_{p i}, & \text { if } T_{i}>T_{m i}\end{cases}
$$

where the specific heat capacity $c_{p i}$ increases linearly with increasing the temperature up to the melting point of a substance, $E_{0 x i}$ is the "cold" component of the specific internal energy, $T_{m i}$ is the melting temperature, $\Delta H_{m i}$ is the specific melting heat of the $i$-th component.

For the mathematical description of explosive compaction, it is necessary to know not only the detonation velocity, but also the pressure of explosion products acting on the steel impactor versus time. In this work, the parameters for the explosive compaction of a cylindrical ampoule containing the $\mathrm{Al}-\mathrm{S}$ mixture are determined upon the assumption [6-8] that the pressure of detonation products $(P)$ linearly decreases with time $t$ :

$$
P=P_{0}-k t,(t=0 \ldots \Delta t)
$$

The coefficient $k$ is given by:

$$
k=\frac{P_{0}}{\Delta t} .
$$

Then the final equation for calculating the pressure of the explosion products takes the form:

$$
P=\left\{\begin{array}{l}
P_{0}-\frac{P_{0}}{\Delta t} t ; 0 \leq t<\Delta t \quad \Delta t=\frac{\Delta}{c}, \quad c=\frac{D}{2}, \\
0 ; \quad t \geq \Delta t,
\end{array}\right.
$$

where $\Delta$ is the thickness of the explosive layer, $c$ is the average velocity of the unloading wave (assessment), $D$ is the detonation velocity. $P_{0}$ is varied in computations to obtain good agreement between numerical and experimental results. For $D=3.3 \mathrm{~km} / \mathrm{s}, P_{0}$ was found to have a value of 3.8 GPa.

\section{RESULTS AND DISCUSSION}

We experimentally investigated the explosive compaction of a cylindrical ampoule that contains a three-component mixture from aluminum, sulfur and graphite. To conduct explosive compaction, the inert substance (graphite) was added to the mixture of aluminum and sulfur in a proportion of
$2 / 1$, where graphite had two mass fractions and the mixture of $\mathrm{Al}-\mathrm{S}$ had one mass fraction to avoid the reaction between aluminum and sulfur. The mass fractions of the components in the sample (mixture) were as follows: $\mathrm{Al}-11.5, \mathrm{~S}-21.5, \mathrm{C}$ 67; volume fractions: $\mathrm{Al}-9.55, \mathrm{~S}-23.35, \mathrm{C}-67.1$. The components were mixed in an AGO-2U planetary mill, and then the mixture was pressed into eight tablets with a diameter of $14 \mathrm{~mm}$ and thickness of $8 \mathrm{~mm}$. The measured porosity was $0.393 \pm 0.005$. The tablets were placed into a steel ampoule with an inner diameter of $14 \mathrm{~mm}$, an external diameter of 20 $\mathrm{mm}$ and a length of $84 \mathrm{~mm}$. The ampoule was closed with lids (Fig. 1a).
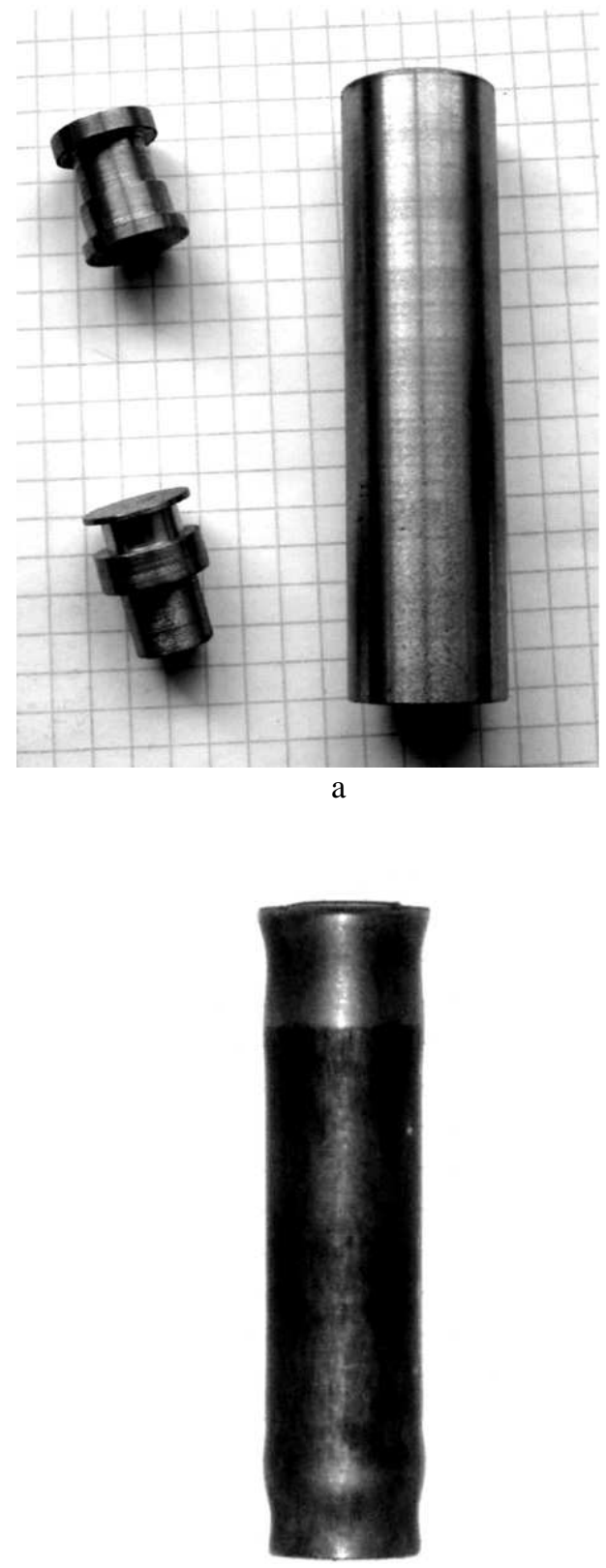

b

Fig.1. Ampoule (a) before and (b) after an experiment. 
The ampoule was loaded by an explosive representing a composition of ammonite 21/79 (TNT/ammonium nitrate) + $\mathrm{NaCl}$ in the proportion of $1 / 1$ by mass. The measured explosive density was $1.2 \mathrm{~g} / \mathrm{cm}^{3}$. The external explosive diameter was $50 \mathrm{~mm}$. The measured detonation velocity was $2.8 \mathrm{~km} / \mathrm{s}$. Fig. 1b shows the ampoule after explosive loading. It is seen that the ampoule is uniformly compressed from top to bottom after the experiment. After comparison of the diameter of the ampoule before and after explosive loading, the compaction degree of the sample was $\approx 97 \%$ (residual porosity was 0.03 ).

To determine the phase composition of the sample, the ampoule was cut with an interval of $1 \mathrm{~cm}$. Fig. 2a shows the upper part of the sample after explosive loading. Composition of the mixture after loading was a homogeneous mixture of Al-S-C. The phase composition in this part of the ampoule was not changed. Approximately $15 \mathrm{~mm}$ from the upper part of the sample, there was the formation of the central zone different in color and, correspondingly, the phase composition from the peripheral part (Fig. 2b). The diameter of the central zone increases towards the lower part of the sample (Fig. 2c).

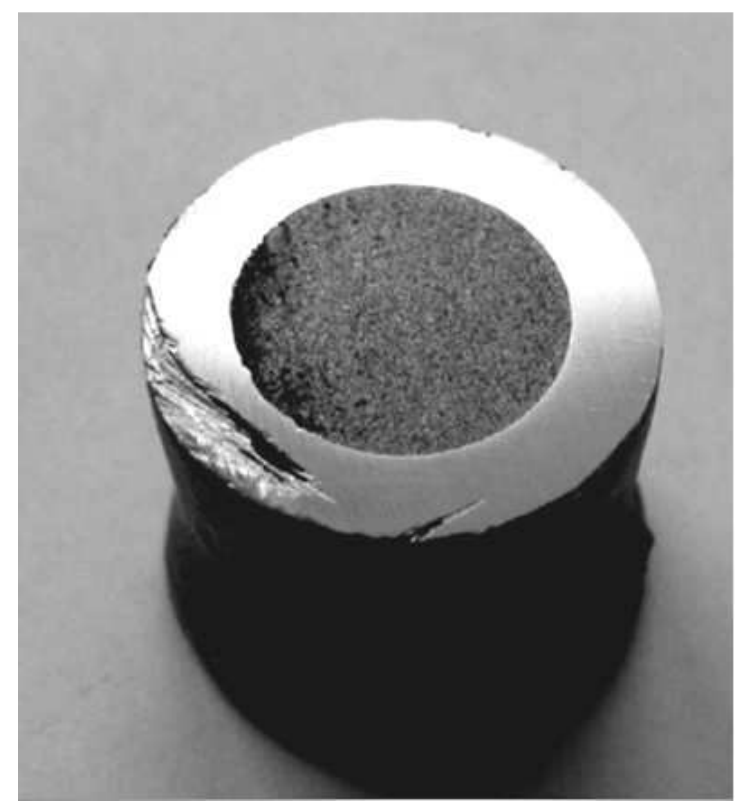

a

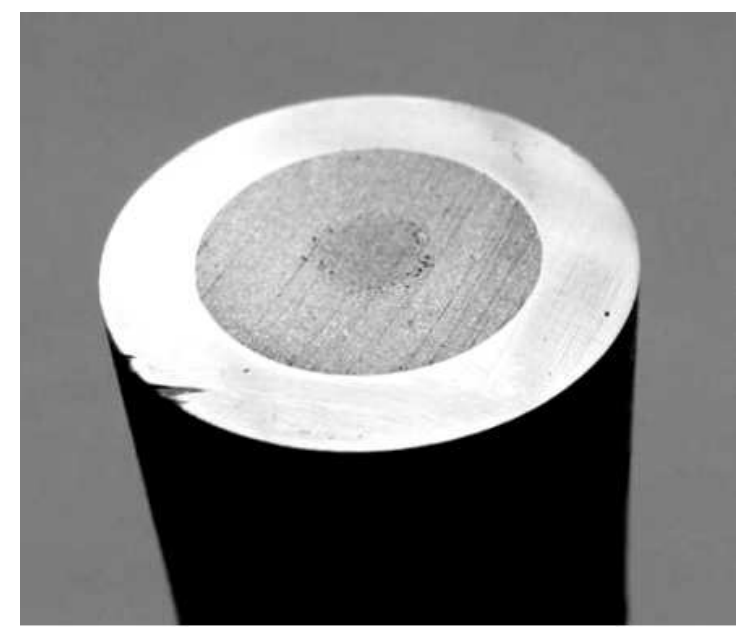

b

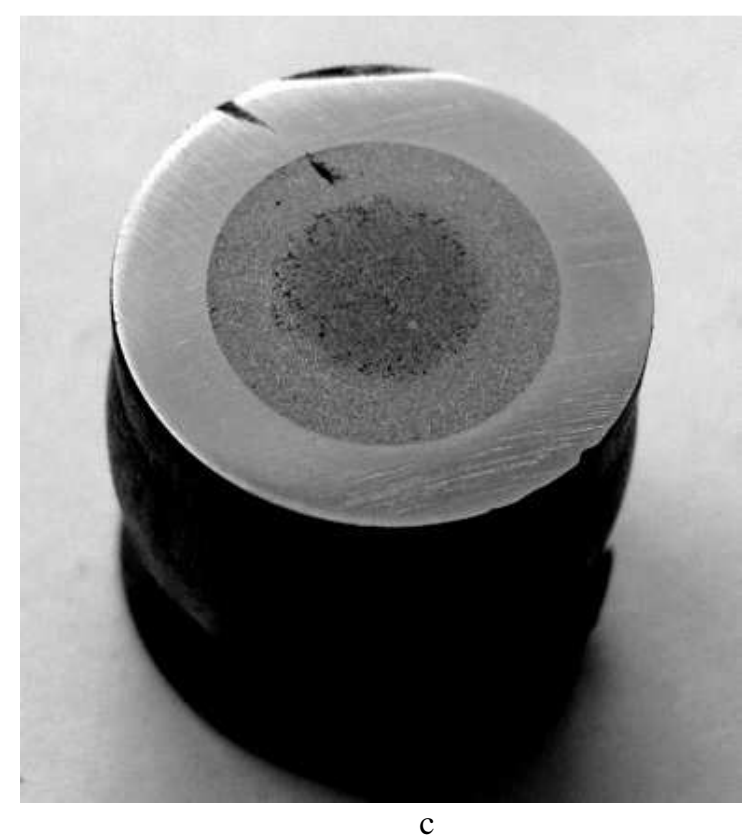

Fig.2. Images of the (a) upper, (b) middle and (c) lower parts of the sample after explosive compaction.

Phase analysis of the material from a central zone showed that it also comprised the reaction products of aluminum and sulfur (phase of aluminum sulfide). In this case, we can draw the conclusion that, despite the addition of graphite to prevent interaction between $\mathrm{Al}$ and $\mathrm{S}$ and reduce their mass fractions in the mixture, $\mathrm{Al}$ and $\mathrm{S}$ partially reacted in the axial region in the middle and bottom parts of the ampoule.

For comparison with the experimental results, we consider the axisymmetric problem of explosive compaction of a threecomponent mixture from aluminum, sulfur and carbon placed into a cylindrical steel ampoule. The inert substance (graphite) was added to the mixture of aluminum and sulfur in a proportion of $2 / 1$ to avoid the reaction between aluminum and sulfur. The mass fractions of the components in the sample (mixture) were taken in proportion: $\mathrm{Al}-11.5, \mathrm{~S}-21.5, \mathrm{C}-$ 67.0; the volume fractions: $\mathrm{Al}-9.55, \mathrm{~S}-23.35, \mathrm{C}-67.1$. The 
porosity of the mixture was 0.4 (ratio between the volume of pores and total volume). The height of the cylindrical sample was $64 \mathrm{~mm}$, the diameter was $14 \mathrm{~mm}$. The thickness of the lateral wall of the ampoule was $3 \mathrm{~mm}$; the thickness of top and bottom lids was $10 \mathrm{~mm}$. The height of the ampoule $(\mathrm{H})$ was 84 $\mathrm{mm}$; the external diameter was $20 \mathrm{~mm}$ (Fig. 3a).

In the computations, the actions of the detonation products surrounding the ampoule was simulated by the action of pressure on the upper part of the ampoule in a vertical (axial) direction and on the lateral surface of the ampoule in a horizontal (radial) direction. In the axial direction, the action started at the initial moment of the process, and in the radial direction, the action stated during propagation of the detonation wave from top to bottom. The detonation velocity was $\mathrm{D}=2.8 \mathrm{~km} / \mathrm{s}$ on the basis of experimental data [10]. The $\mathrm{P}_{0}$ value was chosen on the basis of numerical and experimental evaluations and was $3.2 \mathrm{GPa}$.

In the computations we varied the thickness of the explosive $\Delta_{\mathrm{z}}$ in the axial direction, that act on the upper part of the ampoule in order to study the influence of the parameter on a final shape and size of the ampoule. The value $\Delta_{\mathrm{r}}$ for the explosive acting radially on the lateral wall of the ampoule was constant and equal to $18 \mathrm{~mm}$.

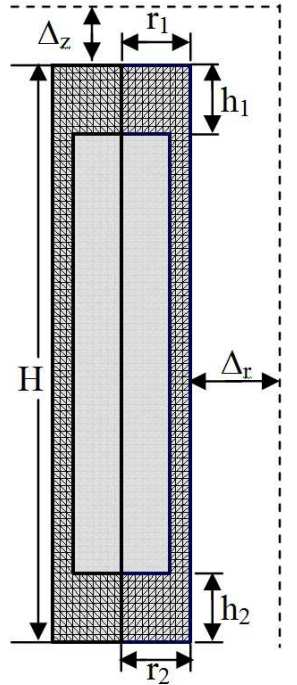

a

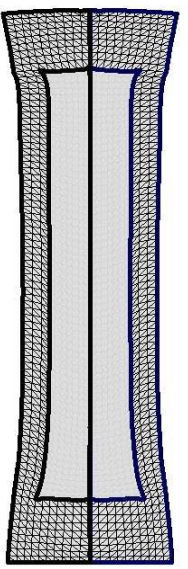

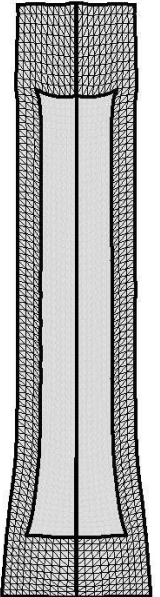

b

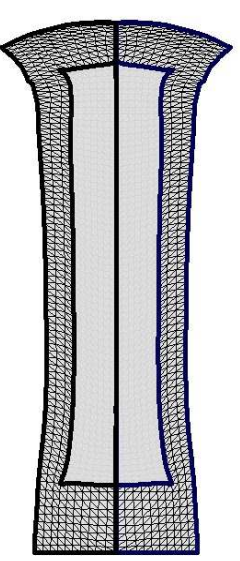

d
Fig. 3. Explosive compaction of the ampoule for different thickness of the axial layer of explosives at the initial moment of time (a) and $80 \mu \mathrm{s}: \Delta_{\mathrm{z}}=5 \mathrm{~mm}(\mathrm{~b}) ; \Delta_{\mathrm{z}}=30 \mathrm{~mm}$ (c); $\Delta_{\mathrm{z}}=40$ $\mathrm{mm}(\mathrm{d})$.

Figs. 3 b-d show the evolution process for the explosive compaction of a cylindrical ampoule with an inert mixture for the different thicknesses of an axial explosive layer at the time of 80 microseconds. This moment of time illustrates the final stage for the explosive compaction of the inert mixture in the cylindrical ampoule. The computations show a significant influence of the axial explosive layer on the final results of explosive compaction. When the thickness $\Delta_{\mathrm{z}}$ is low, the influence of lateral load prevails, which leads to elongation of the ampoule in the axial direction (Fig. 1b). When the thickness $\Delta_{\mathrm{z}}$ is high, there is an additional load on the upper part of the ampoule, which causes deformation of the top lid of the ampoule (compaction in the axial direction and elongation in the radial direction) and a portion of the mixture.

Table 1 shows the numerical results for the loaded cylindrical ampoule with an inert mixture from aluminum, 
sulfur and carbon, when the thickness of the explosive layer is varied in the axial direction. Here $\Delta_{\mathrm{z}}$ is the thickness of the axial layer, $\mathrm{H}$ is the height of the ampoule after loading, $\mathrm{h}_{1}, \mathrm{r}_{1}$ and $h_{2}, r_{2}$ are the height and radius of top and bottom lids of the ampoule. The initial values of the parameters are as follows: $\mathrm{H}=84 \mathrm{~mm} ; \mathrm{h}_{1}=\mathrm{h}_{2}=10 \mathrm{~mm} ; \mathrm{r}_{1}=\mathrm{r}_{2}=10 \mathrm{~mm}$.

TABLE 1. RESULTS OF COMPUTATIONS.

\begin{tabular}{|l|l|l|l|l|l|l|}
\hline № & $\begin{array}{l}\Delta_{\mathrm{z}}, \\
\mathrm{mm}\end{array}$ & $\begin{array}{l}\mathrm{H}, \\
\mathrm{mm}\end{array}$ & $\begin{array}{l}\mathrm{h}_{1}, \\
\mathrm{~mm}\end{array}$ & $\begin{array}{l}\mathrm{h}_{2}, \\
\mathrm{~mm}\end{array}$ & $\begin{array}{l}\mathrm{r}_{1}, \\
\mathrm{~mm}\end{array}$ & $\begin{array}{l}\mathrm{r}_{2}, \\
\mathrm{~mm}\end{array}$ \\
\hline 1 & 0 & 92.8 & 17.2 & 9.0 & 7.6 & 11.1 \\
\hline 2 & 5 & 89.0 & 13.9 & 9.0 & 8.8 & 11.1 \\
\hline 3 & 10 & 85.4 & 11.9 & 9.0 & 9.8 & 11.1 \\
\hline 4 & 15 & 83.2 & 10.8 & 8.9 & 10.3 & 11.1 \\
\hline 5 & 20 & 80.9 & 10.0 & 8.9 & 10.5 & 11.1 \\
\hline 6 & 25 & 78.6 & 9.3 & 8.7 & 11.0 & 11.2 \\
\hline 7 & 30 & 76.1 & 8.2 & 8.7 & 11.9 & 11.2 \\
\hline 8 & 35 & 73.2 & 6.9 & 8.8 & 13.4 & 11.1 \\
\hline 9 & 40 & 69.6 & 5.5 & 8.9 & 15.4 & 11.0 \\
\hline
\end{tabular}

Analyzing the data in Table 1, we can conclude that for positive results of compaction, it is necessary to select a number of parameters for explosive loading. It is important to choose appropriate explosives and the thickness of explosives. Insufficient thickness of explosives, as well as excessive thickness will lead to unsatisfactory results of explosive compaction, in particular, the incompletely compacted final product, cracks and damages. In addition, the excessive thickness of explosives in the axial direction strongly distorts the shape of the ampoule during explosive compaction.

Figs. 4 and 5 show the plots for the parameters of the ampoule with an inert mixture subjected to explosive compaction versus the thickness of the axial explosive layer for the moment of time $80 \mu \mathrm{s}$ after the beginning of the compaction process. The computations show that the wave and deformation processes are completed by this moment of time, and the obtained data can be considered to be the final data after loading.

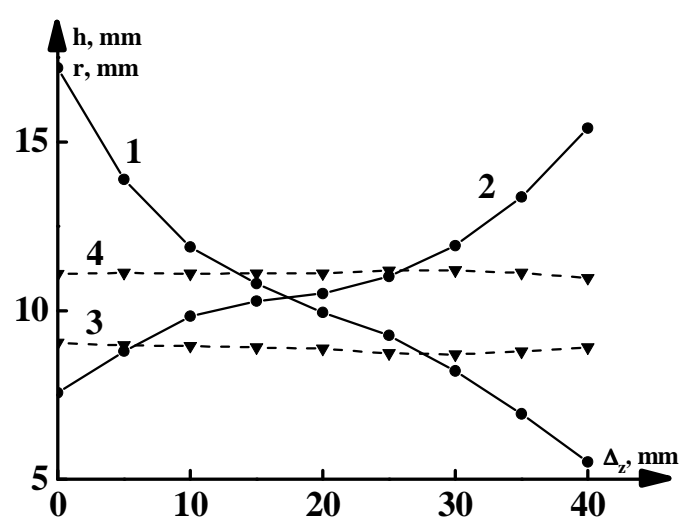

Fig. 4. Height and radius of the ampoule lids versus the thickness of the axial explosive layer: curves 1,2 - height and radius of the upper lid of the ampoule, curves 3, 4 height and radius of the bottom lid of the ampoule.

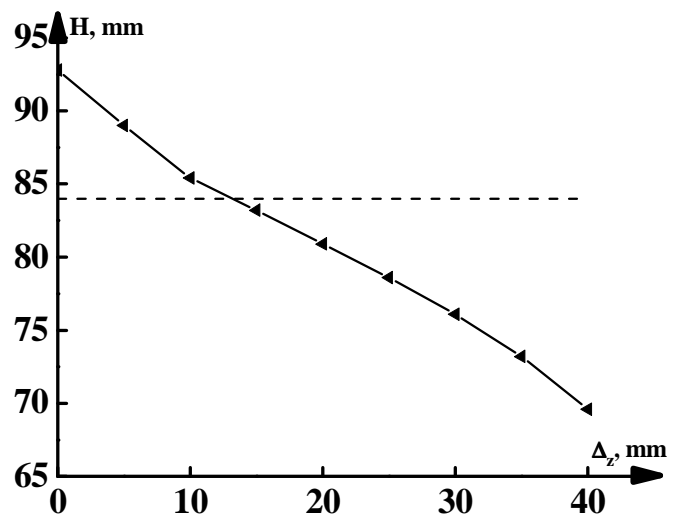

Fig. 5. Height of the ampoule after loading versus the thickness of the axial explosive layer. Dashed line is the initial value of the height of the ampoule.

The computation results show that the change in thickness of the layer $\Delta_{\mathrm{z}}$ within the range of $0 \div 13 \mathrm{~mm}$ is not sufficient for compaction of the ampoule in the axial direction and leads to an increase in height of the ampoule. Using the thickness of the axial explosive layer within the range of $35 \div 40$ leads to a strong distortion of the shape of the ampoule, in particular, the top lid of the ampoule and the mixture in this area. The parameters of ampoule, obtained for the thickness of the axial explosive layer $\Delta_{\mathrm{z}}=30 \mathrm{~mm}$, have shown that in this case the degree of compaction for the sample of the mixture was about 97\%. The parameters for the bottom lid of the ampoule changed insignificantly for all cases.

\section{CONCLUSION}

We experimentally and numerically investigated the explosive compaction of the cylindrical ampoule that contains a solid three-component mixture of aluminum, sulfur and 
graphite. The inert substance (graphite) was added to the mixture to avoid the reaction between aluminum and sulfur.

We studied the influence of the initial thickness of the axial explosive layer on the final shape of the ampoule. We found the essential influence of the thickness of the explosive layer on the final result of explosive compaction. Insufficient thickness of explosives, as well as the excessive thickness may be a reason for an incompletely compacted final product or lead to the formation of cracks or damage.

\section{References}

[1] D.E. Eakins, N.N. Thadhani, Shock Compression of Reactive Powder Mixtures, Int. Mater. Rev. Vol. 54(4), 181-213, 2009.

[2] R. Prümmer, Explosive Compaction of Powders: Principle and Prospects, Materialwiss. Werkstofftech, 20(12), 410-415, 1989.

[3] V.A. Gorelskii, S.A. Zelepugin, S.S. Nabatov, N.B. Nikulichev, Experimental and Numerical Studies of the Behavior of the TitaniumSilicon System Pressurized by Shock Waves in Recovery Ampoules, Chemical Physics Reports, 18(5), 991-1001, 1999.
[4] S.A. Zelepugin, V.B. Nikulichev, Numerical Modeling of Sulfur Aluminum Interaction under Shock-Wave Loading, Combustion, Explosion and Shock Waves, Vol. 36(6), 845-850, 2000.

[5] O. V. Ivanova and S. A. Zelepugin, Explosive Solid-State Synthesis in the Al-S System: Influence of Dispersity and Duration of Shock Loading // International Journal of Self-Propagating High-Temperature Synthesis 23(4) (2014) 191-196.

[6] O. Ivanova, S. Zelepugin, A. Yunoshev, V. Silvestrov, A Multicomponent Medium Model for Reacting Porous Mixtures under Shock Wave Loading, J. Energ. Mater., 28(1), 303-317, 2010.

[7] R.I. Nigmatulin, Dynamics of Multiphase Media, first ed. (Hemisphere, New York, 1991)

[8] G.I. Kanel, V.E. Fortov, S.V. Razorenov, Shock Waves in CondensedState Physics, Physics-Uspekhi, 50(8), 771-791, 2007.

[9] S.A. Zelepugin, O.V. Ivanova, A.S. Yunoshev, V.V. Sil'vestrov, The Development of the Aluminum Sulfide Synthesis Reaction on Explosive Loading of a Cylindrical Ampoule, Doklady Physical Chemistry, 434 (2), 172-176, 2010.

[10] L.B. Pervukhin, M.I. Alymov, I.V. Saikov, R.D. Kapustin, O.L. Pervukhina, E.V. Petrov, Explosive compaction of ceramic powders, Letters on materials, 5(1), 57-60, 2015. 